\title{
Lack of chemopreventive efficacy of metformin in rodent models of urinary bladder, head and neck, and colon/intestine cancer
}

\author{
MATTHEW D. THOMPSON ${ }^{1}$, RONALD A. LUBET $^{2}$, DAVID L. MCCORMICK ${ }^{3}$, MARGIE L. CLAPPER $^{4}$, \\ ANN M. BODE ${ }^{5}$, M. MARGARET JULIANA ${ }^{6}$, FARIBA MOEINPOUR ${ }^{6}$ and CLINTON J. GRUBBS ${ }^{6}$ \\ ${ }^{1}$ Cancer Prevention Fellowship Program; ${ }^{2}$ Chemopreventive Agent Development Research Group, \\ Division of Cancer Prevention, National Cancer Institute, Bethesda, MD 20892; \\ ${ }^{3}$ Life Sciences Group, IIT Research Institute, Chicago, IL 60616; ${ }^{4}$ Division of Population Science, \\ Fox Chase Cancer Center, Philadelphia, PA 19111; ${ }^{5}$ Hormel Institute, University of Minnesota, Austin, \\ MN 55912; ${ }^{6}$ Department of Surgery, University of Alabama at Birmingham, Birmingham, AL 35294, USA
}

Received June 7, 2016; Accepted December 21, 2016

DOI: $10.3892 / 01.2017 .6632$

\begin{abstract}
Metformin is a biguanide employed in treating type II diabetes. Its potential efficacy for treating cancer has been demonstrated epidemiologically (lower cancer incidence in metformin users compared with users of sulfonylureas or insulin) and mechanistically, primarily in cell culture. Metformin decreases the levels of insulin-like growth factor 1 and secondarily inhibits the mammalian target of rapamycin pathway to exhibit anticancer effects. The current study examined its cancer preventive efficacy in multiple standard in situ arising cancer models. Metformin was administered orally by gavage or in the diet, at human equivalent doses, in numerous cancer models. In the hydroxybutyl(butyl)nitrosamine-induced model of invasive urinary bladder cancer, metformin (50 or $150 \mathrm{mg} / \mathrm{kg}$ body weight/day, intragastric) was ineffective despite high urinary concentrations of metformin. Metformin ( 250 or $500 \mathrm{ppm}$ in diet) failed to decrease the incidence or invasiveness of squamous cell cancer of the tongue in a 4-nitroquinoline-1-(4NQO)-induced model. Finally, in the Min mouse model of gastrointestinal cancer, metformin (400 or 1,200 ppm in diet) was ineffective. Notably, a slight increase in intestinal tumor multiplicity was observed at the higher dose. Therefore, metformin lacked efficacy in multiple standard cancer models in non-diabetic rodents. This lack of efficacy may discourage any large phase clinical cancer trials in non-diabetic individuals in the absence of clear phase-II studies.
\end{abstract}

Correspondence to: Dr Clinton J. Grubbs, Department of Surgery, University of Alabama at Birmingham, VH-G78B, Box 8001720 2nd Avenue S., Birmingham, AL 35294, USA

E-mail: clintongrubbs@uabmc.edu

Key words: metformin, prevention, head and neck cancer, colon cancer, urinary bladder cancer

\section{Introduction}

Metformin has been employed as an anti-diabetic agent for a number of years. There have been a number of studies on the use of this agent for cancer chemoprevention or therapy; epidemiological findings suggest a lower incidence of cancer in diabetics receiving metformin compared with diabetics receiving sulfonylurea or insulin $(1,2)$. However a more recent epidemiologic metanalysis which corrects for specifics of metformin diabetic patients fails to confirm any efficacy of metformin (3).

Metformin has a number of potential mechanisms of action through which it may impact the process of carcinogenesis. Two major pathways are: i) Activation of adenosine triphosphate (AMP)-activated protein kinase, which is downstream of metformin effects on the mitochondria, and increased levels of liver kinase B1 (LKB1) $(4,5)$, which may secondarily affect the expression of a wide variety of genes involved in gluconeogenesis and the mammalian target of rapamycin (mTOR) pathway; and ii) reduction in the levels of insulin-like growth factor-1 (IGF1), which may be relevant for a variety of forms of cancer (6).

Although there have been hundreds of studies discussing the use of metformin for cancer prevention, the majority of these have involved cell culture studies (5,7). In fact, in vivo animal data employing in situ cancer models are limited. For this reason, a series of previous studies examined the efficacy of metformin as a chemopreventive agent in animal carcinogenesis models that have been used widely to screen for chemopreventive agents. In our previous study (8), it was demonstrated that, despite altering the pharmacodynamic biomarkers of drug action, metformin was ineffective in preventing estrogen receptor $(\mathrm{ER})^{+}$or ER' types of mammary cancer in two widely used animal models. A similar lack of metformin activity was observed in a third breast cancer model (9). In the present study, the chemopreventive efficacy of metformin was examined in: i) The 4-hydroxybutyl(butyl) nitrosamine (OH-BBN)-induced model of urinary bladder cancer (10) in rats, a model that has significant molecular congruity with invasive bladder cancer in humans (11); 
ii) the 4-nitroquinoline-1-oxide (4-NQO)-induced model of oral squamous cell carcinoma (OSCC) in rats, a model that displays numerous molecular similarities with human oral cancer (12,13); and iii) a modified Min mouse model [carrying a germline adenomatous polyposis coli (APC) mutation] (14) which develops multiple adenomas of the small intestine and is used as a model for human familial adenomatous polyposis (FAP). The results revealed that metformin was ineffective as a chemopreventive agent in each of these widely used in situ carcinogenesis models. By contrast, nonsteroidal anti-inflammatory drugs (NSAIDs) and epidermal growth factor receptor (EGFR) inhibitors have been demonstrated to be effective chemopreventive agents in all three models $(10,12,15)$.

\section{Materials and methods}

Ethical approval. All in vivo studies were performed in full compliance with the Animal Welfare Act and United States Public Health Service Policy on Humane Care and Use of Laboratory Animals. Animal experiments were conducted in facilities at the University of Alabama at Birmingham (Birmingham, AL, USA; urinary bladder cancer studies), IIT Research Institute (Chicago, IL, USA; oral cancer studies) or the Fox Chase Cancer Center (Philadelphia, PA, USA; intestinal cancer studies). Prior to the initiation of in vivo studies at any performing site, study protocols were reviewed and approved by the appropriate Institutional Animal Care and Use Committee. All animals were housed 5/cage in a room lighted $12 \mathrm{~h} /$ day and maintained at $22^{\circ} \mathrm{C}$.

Rat urinary bladder cancer model. This model has been previously described $(10,16)$. The carcinogen $\mathrm{OH}-\mathrm{BBN}$ was purchased from TCI America, Inc. (Portland, OR, USA). OH-BBN (150 mg/gavage) was administered 2 times/week for 8 weeks, beginning when the female Fischer-344 rats ( $n=30 /$ group) were purchased from Envigo (Indianapolis, IN) at 56 days of age. The carcinogen was administered in $0.5-\mathrm{ml}$ ethanol:water $(25: 75, \mathrm{v} / \mathrm{v})$. Two weeks following the last dose of $\mathrm{OH}-\mathrm{BBN}$, the animals received metformin (50 or $150 \mathrm{mg} / \mathrm{kg}$ body weight/day), obtained from the National Cancer Institute Chemical Repository, Bethesda, Maryland, USA) in saline or a saline control until the end of the study. All animals received a Teklad (4\%) mash diet (Envigo, Indianapolis, IN, USA). Animals were observed daily, weighed weekly and palpated for urinary bladder tumors 2 times/week. Rats were sacrificed when they developed a large palpable bladder lesion or were observed to have bloody urine. At necropsy, urinary bladders were inflated with $10 \%$ buffered formalin. Following fixation, the bladder was observed under a high-intensity light for gross lesions. Each lesion was dissected and processed for pathological classification. For immunofluorescence, bladder cancers were fixed in formalin for $24 \mathrm{~h}$ and the switched to $70 \%$ ethanol. After histological processing, the blocks were sectioned at 5 microns and forwarded to the laboratory of Dr. A. Bode for further analyses. The multiplicity and weight of the urinary bladder tumors were determined at the end of the study. Statistical analysis of bladder tumors between groups was performed by the Wilcoxon rank-sum test and the final cancer weights by the Fischer's exact test. The method for determination of metformin concentration in urine was described in our previous study (8).

Urinary bladder immunofluorescence. Paraffin embedded bladder cancer tissue samples on slides were baked in a $60^{\circ}$ oven for $2 \mathrm{~h}$. Paraffin was removed using 4 changes of xylene at 5 min Rehydration of the samples was performed using decreasing percentages of ethanol: three changes of $100 \%$ at $5 \mathrm{~min}$ each, and 5-10 min using the following percentages respectively: 95, 90, 70\%, and DI Water. Final rinse was performed using 1xPBS 2x3 min.

Antigen retrieval was performed using $10 \mathrm{mM}$ sodium citrate buffer ( $\mathrm{pH}$ 6.0). Slides were heated in a 1,200 W microwave for $10 \mathrm{~min}$, allowing to cool at room temperature for 20 min afterward. Rinse slides with DI water for $2 \times 3$ min and then $1 \times$ PBS $2 \times 3$ min.

Specimens were blocked using $10 \%$ normal donkey serum (cat. no., 017-000.121; lot \#121615 Jackson Immuno Research Laboratories, Inc). 1x TPBS for $1 \mathrm{~h}$ at room temperature. Primary antibodies were diluted and samples were labeled as follows: Anti-p53 (total; monoclonal; cat. No. 2524; lot \#4; Cell Signaling Technology Inc., Danvers, MA, USA) used at 1:100 dilution, and anti-phosphorylated (p)-p53 (serine 392; goat; cat. no., sc-7997; lot \#L1809; Santa Cruz Biotechnology, Inc., Dallas, TX, USA) used at 1.25 dilution, and anti-p-p53 (serine 20; goat; cat. no., sc-18078; lot \#C0510; Santa Cruz Biotechnology, Inc.) used at 1.50 dilution. Antibodies were left on overnight at $4^{\circ} \mathrm{C}$.

Samples were rinsed with 1 xPBS. Secondary antibodies were all diluted are 1:200 using 5\% normal donkey serum-1xTPBS and added as follows: anti-cyanine (Cy) 3 (Goat cat. no., 705-165-147; lot \#110658; Jackson Immuno Research Laboratories, Inc.) for p-p53 (ser20), anti-cyanine (Cy) 3 (mouse; cat. No., 715-166-151; lot \#102107; Jackson Immuno Research Laboratories Inc.) for p53 Total and anti-cyanine (Cy) 2 (goat; cat. no., 705-225-147; lot \#103215; Jackson Immuno Research Laboratories, Inc.) for p-P53 (s392). Secondary antibodies were left on for $1 \mathrm{~h}$ and $45 \mathrm{~min}$ and then rinsed with 1xPBS followed by $1 \times 5$ min $1 x$ PBS High Salt (23.38 g NaCl in 1 liter 1x PBS). Coverslip were put on slides using Fluoro-Gel II Dapi (cat. no., 17985-50; lot \#130218; Electron Microscopy Sciences) and seal using clear nail polish. The integrated optical density was calculated as pixel area $x$ mean density. The units listed on the Image-Pro Premier program (Media Cybernetics, Offline Version 9.0, S/N 050900000-1104) are lum/pix ${ }^{2}$.

Rat oral cancer model. We have previously described the rat oral cancer model $(12,13,15)$. Male Fischer 344 rats ( $n=30 /$ group) were obtained from Envigo and were placed into quarantine for a minimum of 1 week. Beginning at 8 weeks of age, rats were exposed to drinking water containing $20 \mathrm{ppm}$ 4-NQO (Sigma-Aldrich; Merck Millipore, Darmstadt, Germany) for a period of 10 weeks. Rats were administered a diet of Purina 5001 Laboratory diet. Dietary administration of metformin (250 or 500 ppm) was initiated 1 day following the final administration of 4-NQO. Alternatively, control rats were administered standard Purina diet without Metformin. In addition, the protocol included a delayed administration group that received $500 \mathrm{ppm}$ metformin beginning 6 weeks 
upon completion of 4-NQO administration. Animals were monitored twice daily for general health status and were weighed weekly. Animals were euthanized by $\mathrm{CO}_{2}$ overdose if they demonstrated a large exophytic oral lesion or lost weight for two successive weeks; the remaining rats were euthanized 14 weeks following the last administration of 4-NQO. All rats underwent a gross necropsy that focused on the tongue and oral cavity. The tongues from each animal were excised and grossly visible oral lesions were charted. Tongues were then bisected longitudinally. Gross lesions and phenotypically normal oral tissues were dissected from one half of each tongue, and were frozen in liquid nitrogen for molecular analyses. The remaining half of each tongue was fixed in $10 \%$ neutral buffered formalin, processed by routine histological methods, and stained with hematoxylin and eosin (H\&E) for pathological classification (12). Cancer invasion was classified using a semiquantitative grading system: Lesions scored as +1 extended through the mucosal epithelial basement membrane and into the lamina propria only; lesions scored as +2 extended through the lamina propria and into the upper muscle layers; and lesions with the highest invasion score of +3 demonstrated extensive infiltration into underlying muscle. Evidence of metformin activity was defined as a statistically significant $(\mathrm{P}<0.05)$ reduction in oral cancer incidence, reduction in oral cancer invasion score or increase in survival in a group treated with metformin vs. that in the carcinogen-treated dietary control group. Inter-group comparisons of oral cancer incidence and survival at the termination of the study were performed using $\chi^{2}$ analysis. As oral cancer invasiveness was evaluated using a semiquantitative scoring system, comparisons of invasion scores were performed using the nonparametric Wilcoxon rank-sum analysis. Body weights and other continuous data were compared using analysis of variance, with post hoc comparisons conducted using the Dunnett's test.

Mouse intestinal tumor model. The methods used for this modified Min mouse assay have been previously described $(14,16)$. Male Apc ${ }^{+/ \text {Min-FCCC }}$ mice (n=10/group, 8 weeks old) were obtained from the Laboratory Animal Facility at Fox Chase Cancer Center. Apc ${ }^{+/ \mathrm{Min}-\mathrm{FCCC}}$ mice demonstrate increased numbers of colorectal adenomas (16) compared with $\mathrm{Apc}^{+/ \mathrm{Min}}$ mice. Animals were acclimated to a modified American Institute of Nutrition-76A semi-purified diet for 1 week prior to starting treatment with metformin. At 9 weeks of age, mice were randomized to receive the control diet or a diet supplemented with 400 or $1,200 \mathrm{ppm}$ metformin. Body weights were recorded weekly to monitor toxicity. Animals were maintained on the experimental diets for 45 days.

At study termination, mice were euthanized $\left(\mathrm{CO}_{2}\right)$ and the entire small intestine was excised, divided into three equal sections (proximal, middle and distal), opened lengthwise and rinsed with PBS. Gross lesions were counted and recorded. Each segment of the small intestine was jelly-rolled, fixed in neutral buffered formalin and embedded entirely in paraffin. One slide of each intestinal segment (5 micron sections) was stained with H\&E (and evaluated pathologically in a blinded manner. Adenomas were defined as circumscribed neoplasms composed of tubular and villous structures and lined with dysplastic epithelium. Cancer tissues were required to meet the following three criteria: i) Invasion into at least the submucosa; ii) able to elicit a desmoplastic reaction; and iii) exhibition of cytological features of neoplasia.

Body weights and tumor multiplicities were compared among treatment groups using the Wilcoxon rank-sum test [NCSS Statistical Software (Salstat, 3rd addition): Wilcoxon Rank Sum Test]. The P-values of the pair-wise comparisons were adjusted using the Bonferroni multi-comparison correction.

\section{Results}

Efficacy evaluation of metformin in the $\mathrm{OH}-\mathrm{BBN}$ rat urinary bladder cancer model. Metformin was administered to rats daily by gavage at 50 or $150 \mathrm{mg} / \mathrm{kg}$ body weight, beginning 2 weeks after the last dose of OH-BBN. When compared with $\mathrm{OH}-\mathrm{BBN}$-treated rats receiving vehicle only, neither dose of metformin reduced bladder cancer incidence or increased bladder cancer latency $(\mathrm{P}>0.05$ for the two comparisons; Fig. 1A). Similarly, neither dose of metformin decreased the final incidence of large palpable cancer. Furthermore, the mean bladder weight, a surrogate measure of tumor weight, did not differ between groups ( $\mathrm{P}>0.05$; Fig. 1B). This lack of chemopreventive activity against urinary bladder carcinogenesis was observed despite the fact that concentrations of metformin in the urine were markedly elevated compared with the concentrations in plasma (data not shown). The doses of metformin employed did not alter final body weights (vehicle, $303 \mathrm{~g}$; high dose metformin, $290 \mathrm{~g}$; low dose metformin, $293 \mathrm{~g}$ ).

Effects of metformin on p53 phosphorylation in bladder tumor. Control OH-BBN-treated rats bearing palpable bladder tumors were treated with metformin for 5 days. Immunohistochemistry for specific p53 phosphorylation sites revealed that metformin increased the phosphorylation at two separate sites on $\mathrm{p} 53$, serine 15 and serine $392(\mathrm{P}<0.01$ for the two comparisons; Fig. 2). These phosphorylations are considered to be secondary changes downstream of the activation of AMP kinase (17).

Efficacy evaluation of metformin in the 4-NQO rat oral cancer model. Metformin was administered in the diet (vehicle, 250 or $500 \mathrm{ppm}$ ) beginning 1 day following the final dose of 4-NQO. An additional group of rats was exposed to metformin $(500 \mathrm{ppm})$ beginning 6 weeks after 4-NQO administration. The final incidence of OSCC in $\mathrm{OH}-\mathrm{BBN}$-treated rats receiving vehicle only was $73 \%$ (22/30 rats). In comparison, OSCC incidences in rats receiving 4-NQO and metformin were $77 \%$ (23/30; low-dose metformin), 73\% (22/30; high-dose metformin) and 67\% (18/27; high-dose metformin, delayed administration); none of these incidences was significantly different from vehicle controls. When compared with vehicle controls, metformin also failed to alter the invasion score of induced OSCC ( $\mathrm{P}>0.05$ for all comparisons) and did not alter the incidence of preneoplastic lesions (squamous cell papillomas or squamous cell hyperplasias; $\mathrm{P}>0.05$ for all comparisons; Fig. 3). The doses of metformin employed did not alter the final body weights (vehicle, $24 \mathrm{~g}$; high does metformin, $22.8 \mathrm{~g}$; low dose metformin, $24.1 \mathrm{~g}$ ). 

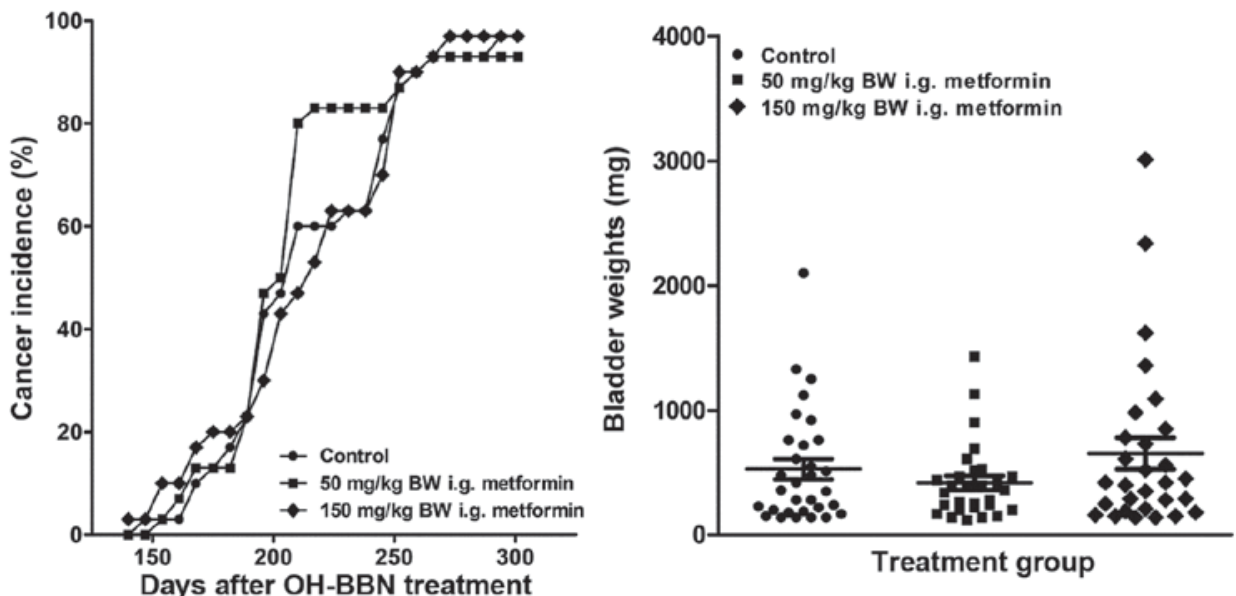

Figure 1. Effect of metformin on urinary bladder cancer incidence, latency and weight. (A) Two varied doses of metformin were administered by gavage (50 or $150 \mathrm{mg} / \mathrm{kg} \mathrm{BW/day)} \mathrm{to} \mathrm{rats} \mathrm{beginning} 2$ weeks after the last dose of OH-BBN. Neither dose affected urinary bladder cancer latency nor decreased the final incidence of large palpable cancers $(\mathrm{P}>0.05)$. (B) Bladder weights, a surrogate measure of tumor weight, did not differ between groups $(\mathrm{P}>0.05)$. $\mathrm{BW}$, body weight; i.g., intragastric; $\mathrm{OH}-\mathrm{BBN}$, 4-hydroxybutyl(butyl)nitrosamine.

Efficacy evaluation of metformin in the Min mouse intestinal tumor model. The survival rate for mice receiving diets supplemented with 400 or 1,200 ppm metformin was $\geq 95 \%$; this survival was comparable to that of the untreated control group. Gross tumor counts in the small intestine (either per segment or total) in mice that were administered a diet containing $400 \mathrm{ppm}$ metformin were comparable to those of untreated control mice ( $\mathrm{P}=0.49$; Fig. 4). Although not statistically significant, animals administered a diet supplemented with $1,200 \mathrm{ppm}$ metformin exhibited a $\sim 2$-fold increase in the mean multiplicity of gross small intestinal tumors, particularly in the mid and distal segments $(\mathrm{P}=0.069$ and $\mathrm{P}=0.097$, respectively; Fig. 4). These results demonstrate non-significant increases in tumor multiplicity in mice receiving the high dose of metformin, and non-significant decreases in tumor incidence in mice receiving the low dose of metformin. In the colon, a dose-dependent increase in tumor multiplicity was observed; however, this trend was not statistically significant $(\mathrm{P}>0.05)$. The doses of metformin employed did not alter final body weights (vehicle, $24 \mathrm{~g}$; high does metformin, $22.8 \mathrm{~g}$; low dose metformin, $24.1 \mathrm{~g}$ ).

\section{Discussion}

As a result of its relatively low toxicity and its ability to alter energy metabolism pathways that are important for neoplastic growth, metformin has been the subject of numerous studies as a potential cancer chemopreventive and therapeutic agent $(1,2,6)$. The results of several early epidemiology studies suggested that individuals taking metformin had a reduced risk of cancer; however, several more recent epidemiologic studies have failed to confirm these findings $(3,17,18)$. It has been proposed that metformin acts directly on mitochondria, which secondarily affects LKB1 and cyclic AMP kinase levels (4), finally altering the mTOR pathway. Additionally, the systemic effects of metformin on IGF-1 signaling may be mediated through the liver. Thus there are multiple direct and indirect mechanisms by which metformin may target cancer cells $(19,20)$. In vitro studies have supported these expected

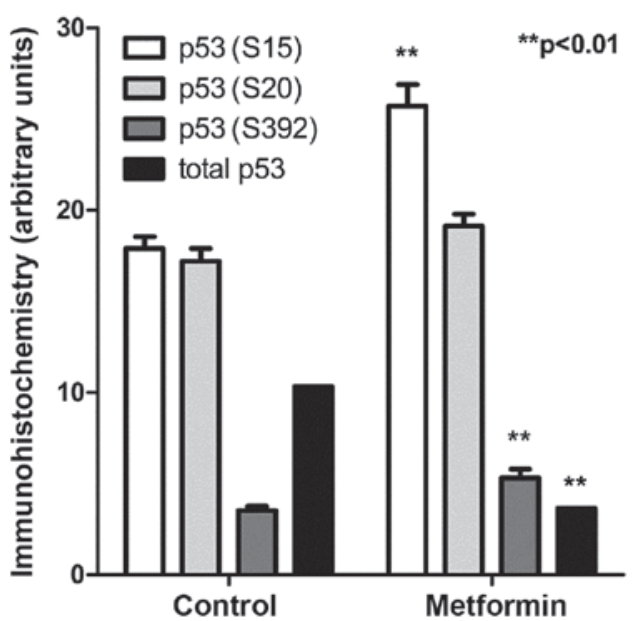

Figure 2. Effect of metformin on p53 phosphorylation in urinary bladder cancer. Immunofluorescence for specific p53 phosphorylation sites indicated that metformin had biological activity in urinary bladder cancer [p53 (serine 15), $\mathrm{P}<0.01$; p53 (serine 392), $\mathrm{P}<0.01]$.

mechanistic results and shown that metformin is preferentially effective in tumor cells (6).

Our previous study reported that metformin was ineffective in preventing neoplastic development in animal models of $\mathrm{ER}^{+}$and ER breast cancer (8), in which metformin provided no significant protection against tumor development in the $\mathrm{N}$-methyl-N-nitrosourea (MNU)-induced $\mathrm{ER}^{+}$mammary cancer model in rats or in the MMTV-Neu/P53KO transgenic mouse model for ER mammary tumors. The results of the present study provide additional chemoprevention efficacy data for metformin in three well-characterized animal models of cancer in organ sites that are not associated with the mammary gland. Metformin failed to provide protection against carcinogenesis in any of the three models tested.

Using standard interspecies scaling factors based on $\mathrm{mg} / \mathrm{m}^{2}$ equivalent doses, the metformin dose of $150 \mathrm{mg} / \mathrm{kg}$ body weight/day was selected for use in our previous $\mathrm{ER}^{+}$ breast cancer chemoprevention study (8) as the rat equivalent 
A

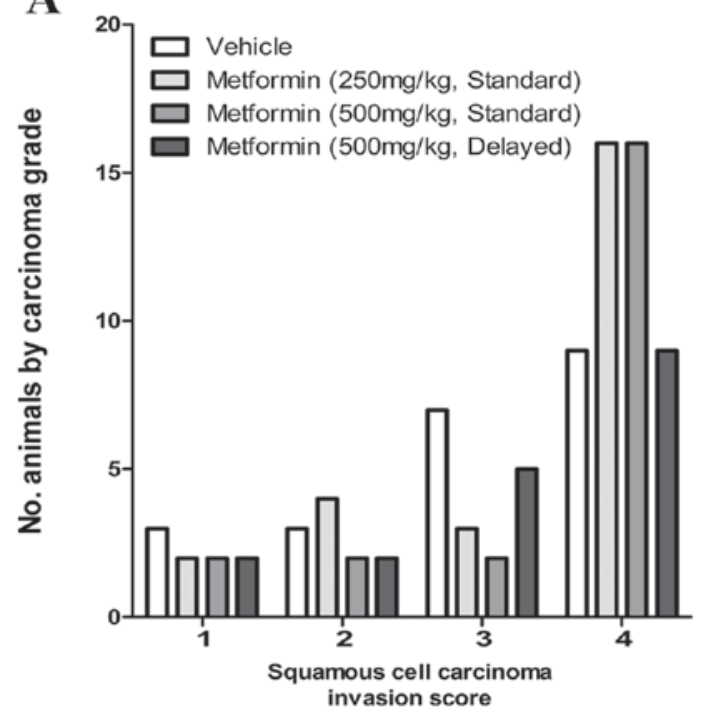

B

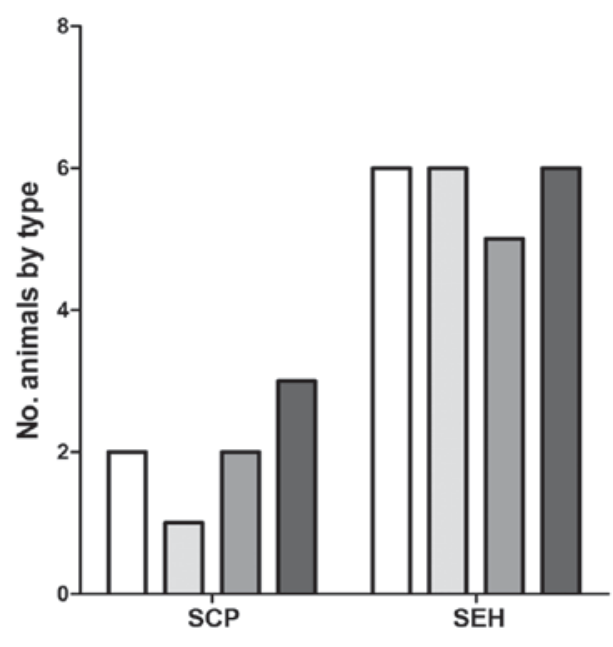

Figure 3. Effect of metformin on the 4-NQO-induced model of squamous cell cancer grade and type. Metformin was administered in the diet (vehicle, 250, 500 or 500 ppm with 6-week delayed intervention) beginning 5 days following the final dose of 4-NQO in drinking water. Metformin (A) failed to alter the incidence of any lesion grade $(\mathrm{P}>0.05)$ and $(\mathrm{B})$ did not alter non-cancerous lesion types (P>0.05). 4-NQO, 4-nitroquinoline-1-oxide; SCP, squamous cell papilloma; $\mathrm{SEH}$, squamous cell epithelial hyperplasia.

of a daily human dose of $\sim 1.5 \mathrm{~g}$. Notably, the Cmax (maximal concentration) and area under the curve (AUC) values of this dose in rats were 2-3-fold greater than the $\mathrm{Cmax}(3.1 \pm 0.9 \mathrm{mg} / \mathrm{ml}$; $20 \mu \mathrm{M})$ and AUC $(18.4 \pm 6.5 \mathrm{mg} / \mathrm{ml} / \mathrm{h})$ values reported for humans. Despite the fact that the metformin Cmax and AUC in rodent models were higher compared with those achieved in humans $(21,22)$, metformin demonstrated no chemopreventive activity in the $\mathrm{ER}^{+}$rat mammary cancer model (8). In this regard, the peak concentration of metformin achieved in serum of rats $(7.5 \mathrm{mg} / \mathrm{l})$ is equivalent to a concentration of $60 \mathrm{mM}(8)$; these concentrations ( $20 \mu \mathrm{M}$ in humans or $60 \mathrm{mM}$ in rats) are notably lower than the metformin concentrations of $2.0 \mathrm{mM}$ that are routinely used in cell culture experiments $(4,5)$.

In the urinary bladder study, metformin concentrations were measured in overnight urine samples collected from vehicle or metformin-treated rats. Using the analytic methods described previously (8) serum levels of $55 \mu \mathrm{M}, 900 \mu \mathrm{M}$ and $2800 \mu \mathrm{M}$ (overnight urines at days 1 and 14) were observed. This reflects the fact that metformin is routinely excreted unmetabolized in urine in rodents and humans. Despite these high urinary concentrations, metformin was ineffective as a chemopreventive agent in the $\mathrm{OH}-\mathrm{BBN}$-induced bladder cancer model.

Parenthetically, our previous studies have reported that tumors in this model have strong molecular congruity with invasive human bladder cancer $(11,23)$. In addition, cyclooxygenase (COX) inhibitors and EGFR inhibitors (10) have been demonstrated to be highly effective in bladder cancer prevention in this rat model, even when their administration is initiated in the presence of microscopic lesions. However, metformin was completely ineffective in the present study. This negative result is somewhat unexpected considering that i) very high levels of metformin were measured in the urine of treated rats and ii) phosphorylation of p53 (serine 15 and serine 392) was demonstrated by immunohistochemistry in urinary

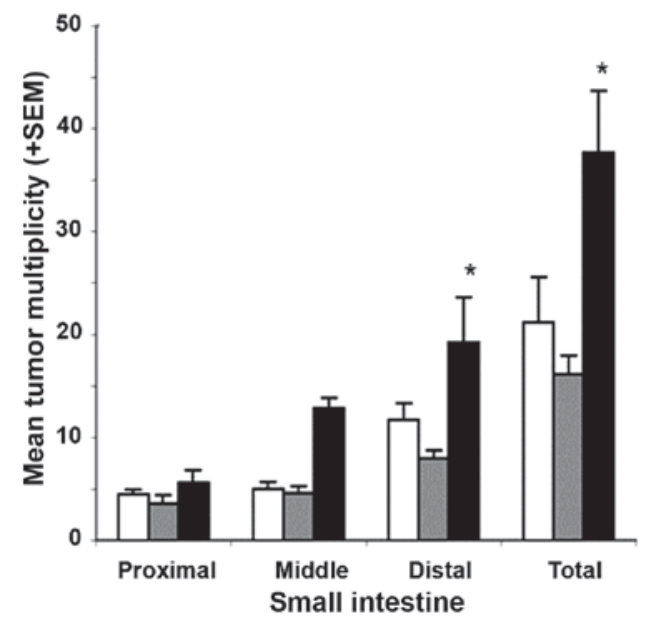

Figure 4. Effect of metformin on the multiplicity of gross small intestinal tumors in $\mathrm{Apc}^{+/ \mathrm{Min}-\mathrm{FCCC}}$ mice. Total represents the sum of lesions in the proximal, mid and distal small intestine. Statistical analyses were performed using the Wilcoxon test. White bars indicate the control diet, gray bars indicate $400 \mathrm{ppm}$ metformin in the diet and black bars indicate 1,200 ppm in the diet. *Significantly different from $400 \mathrm{ppm}$ metformin (total small intestine, $\mathrm{P}<0.03$; distal small intestine, $\mathrm{P}<0.02)$. SEM, standard error of the mean.

bladder cancer tissues in metformin-treated rats. These data suggest that neither metformin pharmacokinetics nor pharmacodynamics were limiting in this model. There have been a number of papers dealing with the use of metformin in bladder models in rodents (24-26). The first two studies required intraperitoneal or intravesicular administration of metformin to achieve efficacy in bladder graft models and therefore are quite unlike the present studies. The results from Liu et al (26) appear to be in greater conflict with the present study. This study was performed in an in situ model of bladder cancer induced by MNU, and metformin was administered to rats in water. The authors argued that the moderate inhibition of 
bladder cancer observed was due to inhibition of prostaglandin E levels secondary to the inhibition of COX-2. However, the model we employed in the current study is profoundly sensitive to COX-2 inhibition (10) but was not inhibited by metformin.

The present study also examined the chemopreventive activity of metformin in the 4-NQO-induced model of OSCC. Oral cancers induced in this model are phenotypically similar to invasive oral cancers, develop at a site that is a common location for human oral cancer (the tongue) and demonstrate significant molecular congruity with human oral cancers $(12,13)$. These highly invasive lesions, although sensitive to the preventive effects of several NSAIDs (12), peroxisome proliferator-activated receptor $\gamma$ agonists (15) and EGFR tyrosine kinase inhibitors (15), were not affected by treatment with metformin. Metformin had no effect on the incidence of OSCC, did not impact the OSCC invasion score and had no effect on the incidence of preneoplastic lesions in this animal model.

Finally, the chemopreventive efficacy of metformin was evaluated in the Min mouse model of intestinal cancer in the current study. Min mice have a germline mutation in the APC gene similar to humans with FAP. Furthermore, mutations in the APC or $\beta$-catenin genes are observed in the preponderance of sporadic colon cancers in mice (14). The Min model, by contrast to human FAP, yields multiple intestinal lesions rather than colonic lesions. As was the case with the urinary bladder cancer and oral cancer studies, metformin failed to confer any protection against intestinal carcinogenesis in the Min mouse model in the present study. Reductions in tumor multiplicity of $60-80 \%$ have been reported in other Min mouse chemoprevention studies with NSAIDs, COX-2 inhibitors and difluoromethylornithine (27). It is of note that one study has reported a positive result for metformin in the Min mouse model (28). However, these investigators identified virtually no decrease in tumor multiplicity, as the reported positive result was based on a small, yet significant, effect on tumor size (28).

Pharmacokinetic data revealing high urinary concentrations of metformin and demonstration of the modulation of a pharmacodynamics endpoint (increased p53 phosphorylation) in the urinary bladder cancer model may suggest potential chemopreventive activity. However, in none of the three models used in the present study did metformin demonstrate a beneficial effect on tumor incidence, multiplicity or growth. The observed lack of correlation between p53 activation and chemopreventive efficacy may be interpreted as suggesting that clinical trials at the phase-IIA level may use biomarkers that are more directly related to preventive efficacy, including cell proliferation or apoptosis $(29,30)$ and not biochemical parameters, including activated AMP kinase, and pharmacokinetic data alone.

In the present study, metformin demonstrated no statistically significant protective activity against neoplastic development in animal models for cancer of the urinary bladder, oral cavity or intestinal tract. The lack of metformin chemopreventive activity in these studies, when considered with our previous data showing lack of chemopreventive efficacy in two mammary cancer models, argues that metformin is not a high-priority candidate for clinical study as a chemopreventive agent in persons without diabetes or exhibiting insulin resistance.
One major caveat regarding these three studies (as well as our previous mammary cancer studies) is that these studies were conducted in non-obese, non-diabetic rodent models. Results may have been altered in diabetic or insulin-resistant animals, in which physiological effects on the underlying disease may have effects on the tumor outcome. A previous study has indicated preferable efficacy for metformin in rodents with diabetes (31). The results of the current study are, nevertheless, relevant to the preponderance of ongoing clinical trials that specifically exclude diabetics and administer metformin orally. A previous metformin biomarker-based study using proliferation as a potential biomarker identified that a large placebo-controlled trial exhibited differences in efficacy based on insulin resistance (32). However, even in this example, the decrease in proliferation in lesions observed in individuals with insulin resistance treated with metformin are not nearly as notable as those routinely induced by selective estrogen receptor modulators or aromatase inhibitors in similar lesions (29). Furthermore, if the majority of the effect occurs in persons with diabetes, then one cannot randomize such individuals to a placebo-controlled trial or even easily develop a trial comparing two alternative anti-diabetic drugs. Future work may be performed in normal and pre-diabetic animals to further interrogate the potential use of metformin for cancer prevention.

\section{Acknowledgements}

The funding for the current study was provided in part by the National Cancer Institute (Bethesda, MD, USA; grant nos. HHSN261200433001C, HHSN261201200021I and HHSN261200433003C).

\section{References}

1. Noto H, Goto A, Tsujimoto T and Noda M: Cancer risk in diabetic patients treated with metformin: A systematic review and meta analysis. PLoS One 7: e33411, 2012.

2. Decensi A, Puntoni M, Goodwin P, Cazzaniga M, Gennari A, Bonanni B and Gandini S: Metformin and cancer risk in diabetic patients: A systematic review and meta analysis. Cancer Prev Res (Phila) 3: 1451-1461, 2010.

3. Gandini S, Puntoni M, Heckman-Stoddard BM, Dunn BK, Ford L, DeCensi A and Szabo E: Metformin and cancer risk and mortality: A systematic review and meta-analysis taking into account biases and confounders. Cancer Prev Res (Phila) 7: 867-885, 2014.

4. Hardie DG, Ross FA and Hawley SA: AMPK: A nutrient and energy sensor that maintains energy homoestasis. Nat Rev Mol Cell Biol 13: 251-262, 2012.

5. Pollak MN: Investigating metformin for cancer prevention and treatment: The end of the beginning. Cancer Discov 2: 778-790, 2012.

6. Pollak M: The insulin and insulin-like growth factor receptor family in neoplasia; An update. Nat Rev Cancer 12: 159-169, 2012.

7. Gallagher EJ and Le Roith D: Diabetes, cancer, and metformin: Connections of metabolism and cell proliferation. Ann N Y Acad Sci 1243: 54-68, 2011.

8. Thompson MD, Grubbs CJ, Bode AM, Reid JM, McGovern R, Bernard PS, Stijleman IJ, Green JE, Bennett C, Juliana MM, et al: Lack of effect of metformin on mammary carcinogenesis in nondiabetic rat and mouse models. Cancer Prev Res (Phila) 8: 231-239, 2015.

9. Zhu Z, Jiang W, Thompson MD, Echeverria D, McGinley JN and Thompson HJ: Effects of metformin, buformin, and phenformin on the post-initiation stage of chemically induced mammary carcinogenesis in the rat. Cancer Prev Res (Phila) 8: 518-527, 2015. 
10. Lubet RA, Steele VE, Juliana MM and Grubbs CJ: Screening agents for preventive efficacy in a bladder cancer model: Study design, end points, and gefitinib and naproxen efficacy. J Urol 183 : $1598-1603,2010$.

11. Lu Y, Liu P, Wen W, Grubbs CJ, Townsend RR, Malone JP, Lubet RA and You M: Cross-species comparison of orthologous gene expression in human bladder cancer and carcinogen-induced rodent models. Am J Transl Res 3: 8-27, 2010.

12. McCormick DL, Phillips JM, Horn TL, Johnson WD, Steele VE and Lubet RA: Overexpression of cyclooxygenase-2 in rat oral cancers and prevention of oral carcinogenesis in rats by selective and nonselective COX inhibitors. Cancer Prev Res (Phila) 3: 73-81, 2010.

13. Peng X, Li W, Johnson WD, Torres KE and McCormick DL: Overexpression of lipocalins and pro-inflammatory chemokines and altered methylation of PTGS2 and APC2 in oral squamous cell carcinomas induced in rats by 4-nitroquinoline-1-oxide. PLoS One 10: e0116285, 2015.

14. Cooper HS, Chang WC, Coudry R, Gary MA, Everly L, Spittle CS, Wang H, Litwin S and Clapper ML: Generation of a unique strain of multiple intestinal neoplasia (Apc(+/Min-FCCC)) mice with significantly increased numbers of colorectal adenomas. Mol Carcinog 44: 31-41, 2005.

15. McCormick DL, Horn TL, Johnson WD, Peng X, Lubet RA and Steele VE: Suppression of rat oral carcinogenesis by agonists of peroxisome proliferator activated receptor $\gamma$. PLoS One 10: e0141849, 2015.

16. Lubet RA, Clapper ML, McCormick DL, Pereira MA, Chang WC, Steele VE, Fischer SM, Juliana MM and Grubbs CJ: Chemopreventive efficacy of Targretin in rodent models of urinary bladder, colon/intestine, head and neck and mammary cancers. Oncol Rep 27: 1400-1406, 2012.

17. Giovannucci E, Harlan DM, Archer MC, Bergenstal RM, Gapstur SM, Habel LA, Pollak M, Regensteiner JG and Yee D: Diabetes and cancer: A consensus report. CA Cancer J Clin 60: 207-221, 2010

18. Evans JM, Donnelly LA, Emslie-Smith AM, Alessi DR and Morris AD: Metformin and reduced risk of cancer in diabetic patients. BMJ 330: 1304-1305, 2005.

19. Jones RG, Plas DR, Kubek S, Buzzai M, Mu J, Xu Y, Birnbaum MJ and Thompson CB: AMP-activated protein kinase induces a p53-dependent metabolic checkpoint. Mol Cell 18: 283-293, 2005.

20. Pollak M: Metformin and other biguanides in oncology: Advancing the research agenda. Cancer Prev Res (Phila) 3 . 1060-1065, 2010.

21. Tucker GT, Casey C, Phillips PJ, Connor H, Ward JD and Woods HF: Metformin kinetics in healthy subjects and in patients with diabetes mellitus. Br J Clin Pharmacol 12: 235-246, 1981.
22. Martin-Castillo B, Vazquez-Martin A, Oliveras-Ferraros C and Menendez JA: Metformin and cancer: Doses, mechanisms and the dandelion and hormetic phenomena. Cell Cycle 9: 1057-1064,2010.

23. Williams PD, Lee JK and Theodorescu D: Molecular credentialing of rodent bladder carcinogenesis models. Neoplasia 10 838-846, 2008.

24. Zhang T, Guo P, Zhang Y, Xiong H, Yu X, Xu S, Wang X, He D and Jin $\mathrm{X}$ : The antidiabetic drug metformin inhibits the proliferation of bladder cancer cells in vitro and in vivo. Intl $\mathbf{J} \mathrm{Mol}$ Sci 14: 24603-24618, 2013.

25. Peng M, Su Q, Zeng Q, Li L, Liu Z, Xue L, Cheng Y, Huang Y, Tao T, Lv H, et al: High efficacy of intravescicular treatment of metformin on bladder cancer in preclinical model. Oncotarget 7: 9102-9117, 2016

26. Liu Q, Yuan W, Tong D, Liu G, Lan W, Zhang D, Xiao H, Zhang Y, Huang Z, Yang J, et al: Metformin represses bladder cancer progression by inhibiting stem cell repopulation via COX2/PGE2/STAT3 axis. Oncotarget 7: 28235-28246, 2016

27. Fischer SM, Hawk ET and Lubet RA: Coxibs and other nonsteroidal anti-inflammatory drugs in animal models of cancer chemoprevention. Cancer Prev Res (Phila) 4: 1728-1735, 2011.

28. Tomimoto A, Endo H, Sugiyama M, Fujisawa T, Hosono K, Takahashi H, Nakajima N, Nagashima Y, Wada K, Nakagama $H$ and Nakajima A: Metformin suppresses intestinal polyp growth in ApcMin/+ mice. Cancer Sci 99: 2136-2141, 2008.

29. Dowsett M, Nielsen TO, A'Hern R, Bartlett J, Coombes RC, Cuzick J, Ellis M, Henry NL, Hugh JC, Lively T, et al: Assessment of Ki67 in breast cancer: Recommendations from the International Ki67 in breast cancer working group. J Natl Cancer Inst 103: 1656-1664, 2011.

30. Hadad S, Iwamoto T, Jordan L, Purdie C, Bray S, Baker L, Jellema G, Deharo S, Hardie DG, Pusztai L, et al: Evidence for biological effects of metformin in operable breast cancer: A pre-operative, window-of-opportunity, randomized trial. Breast Cancer Res Treat 128: 783-794, 2011.

31. Algire C, Amrein L, Zakikhani M, Panasci L and Pollak M: Metformin blocks the stimulative effect of a high-energy diet on colon carcinoma growth in vivo and is associated with reduced expression of fatty acid synthase. Endocr Relat Cancer 17: 351-360, 2010.

32. Bonanni B, Putoni M, Cazzaniga M, Pruneri G, Serrano D, Guerrieri-Gonzaga A, Gennari A, Trabacca MS, Galimberti V, Veronesi $\mathrm{P}$, et al: Dual effect of metformin on breast cancer proliferation in a randomized presurgical trial. J Clin Oncol 30: 2593-2600, 2012 